\title{
Cardiac Phlegmon: Infectious Endocarditis Causing Ventricular Wall Rupture
}

\author{
Felix Fleißner $^{1,2(0)}$ Igor Tudorache ${ }^{1}$ Matthias Christgen ${ }^{3}$ Serghei Cebotari ${ }^{1}$
}

\author{
${ }^{1}$ Department of Cardiac, Thoracic, Transplantation, and Vascular \\ Surgery, Hannover Medical School, Hannover, Germany \\ 2 Institute for Molecular and Translational Therapeutic Strategies \\ (IMTTS), Hannover Medical School, Hannover, Germany \\ 3 Institute of Pathology, Hannover Medical School, Hannover, \\ Niedersachsen, Germany
}

Thorac Cardiovasc Surg Rep 2020;9:e51-e54.

\begin{abstract}
Address for correspondence Felix Fleißner, MD, Department of Cardiac, Thoracic, Transplantation, and Vascular Surgery, Hannover Medical School, Carl-Neuberg- Strasse 1, Hannover 30625, Germany (e-mail: fleissner.felix@mh-hannover.de).
\end{abstract}

\begin{abstract}
Keywords

- open heart surgery

- infection

- aneurysm

Background Rupture of the cardiac ventricular wall is a rare and essentially lethal complication of infectious endocarditis valvularis.

Case Description We report a case of a 49-year-old female patient with infectious endocarditis of the aortic valve. Following aortic valve replacement, the patient developed a sudden free left ventricular wall rupture. Immediate reoperation was successful. Histopathology revealed a myocardial infarction due to septic thromboembolism causing a phlegmonlike myocardial appearance.

Conclusion This is a rare case of a myocardial phlegmon with subsequent cardiac lateral wall rupture in the context of an infectious endocarditis with septic coronary embolism.
\end{abstract}

\section{Background}

Infectious endocarditis valvularis is the inflammation of cardiac valves caused by bacterial or fungal microorganisms, which have entered the bloodstream through dental and other surgical procedures or (micro)traumata. Of note, infectious endocarditis remains a disease with high mortality. Despite the growing armamentarium of antimicrobial therapy and improved surgical intervention possibilities, up to $20 \%$ of patients succumb to their disease within the first 30 days after diagnosis. ${ }^{1-3}$

Rupture of the cardiac ventricular wall is a rare but essentially lethal complication of infectious endocarditis and is usually caused by mitral valve annular abscesses. ${ }^{4}$ Acute heart failure, hemopericardium, and exitus letalis are almost inevitable. ${ }^{4,5}$ Septic thromboembolism of the myocardium is usually limited to spotty myocardial infarctions. ${ }^{6}$ In rare cases, septic thromboembolism can also cause confluent myocardial infarctions. These represent a locus minoris resistentiae and bear the risk of ventricular rupture.
Free left ventricular wall rupture due to mitral valve annular abscesses and left ventricular wall rupture in intravenous drug addicts have been illustrated in recent case reports. ${ }^{4,5}$ These reports were based on postmortem sections. However, development of free ventricular wall myocardial phlegmon with spontaneous rupture as a result of septic coronary embolism and its subsequent successful surgical treatment, to our knowledge, has not yet been reported.

\section{Case Report}

A 49-year-old female was presented to a peripheral hospital after returning home from a vocational visit to Rome, Italy. She complained of fatigue, dizziness, and ntermittent fever. Physical examination revealed a minor scratch on her left foot. The patient stated having had a trauma at her left foot a few days prior to the onset of symptoms. Computed tomography (CT) revealed a large vegetation at the aortic valve cusp and a pericardial effusion (-Fig. 1). The patient was placed on calculated antibiotic therapy and was referred received

December 3, 2019

accepted

January 24, 2020
DOI https://doi.org/

10.1055/s-0040-1716391. ISSN 2194-7635.
๑) 2020 Georg Thieme Verlag KG
Stuttgart · New York

License terms

$(\circledast) \Theta \circledast$ 


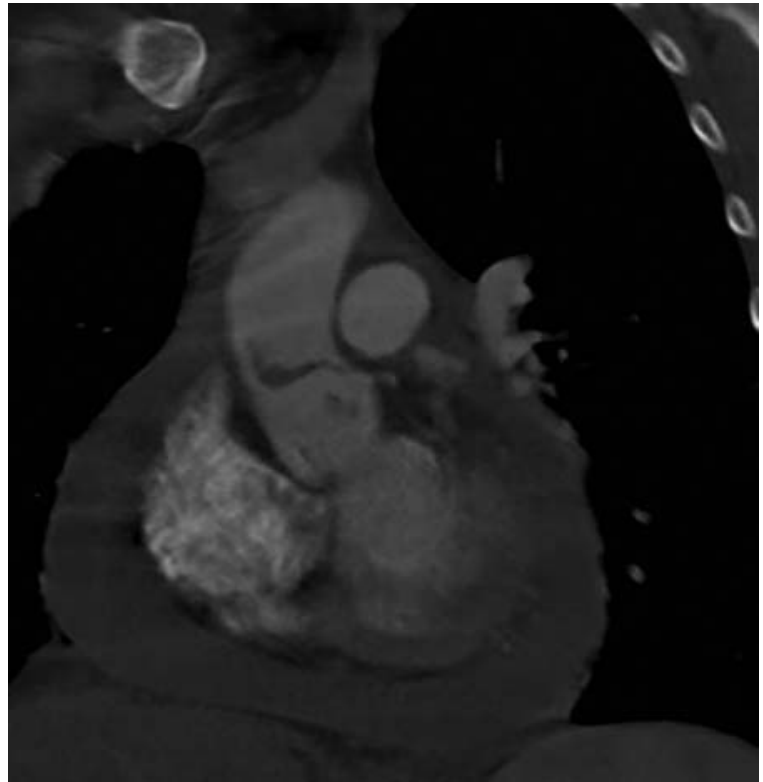

Fig. 1 Computed tomography (CT) of the patient prior to operation. A large vegetation can be seen adjacent to the aortic valve. Pericardial effusion is clearly visible.

to the department of cardiology of a peripheral hospital. Due to the enormous size of the vegetation $(\sim 3 \mathrm{~cm}$ long) and increasing pericardial effusion, she was referred to our center for emergency surgery. After a standard sternotomy, the pericardium was opened and a hemorrhagic pericardial effusion was detected. The patient was placed on cardiopulmonary bypass (CPB). After aortotomy, the large vegetation was identified in close proximity to the left main coronary artery. Great care was taken to prevent thromboembolism during removal of the vegetation. At closer inspection, two small septic perforations of the aortic wall were detected right above the aortic valve annulus. They were directly closed using two Prolene 4-0 sutures (Ethicon Inc., Somerville, NJ). The aortic valve was replaced in standard technique using a 21-mm SJM mechanical valve (St. Jude Medical Inc., Minneapolis, MN). After uneventful weaning of $\mathrm{CPB}$ with no bleeding in situ, the chest was closed and the patient was transferred under stable hemodynamic condition with mild dosages of catecholamines to the intensive care unit (ICU). About an hour after arrival in the ICU, suddenly large quantities of blood $(1.5 \mathrm{~L})$ were detected in the thoracic drainage. The patient became hemodynamic unstable, and a reoperation was immediately initiated. On the way to the operation room, the patient needed resuscitation. In the operation room, the sternum was reopened under resuscitation and the patient was placed on CPB. After preliminary hemodynamic stabilization, inspection of the pericardium revealed a large rupture of the left ventricular lateral wall $(1.5 \times 1.5 \mathrm{~cm}$ size $)(-$ Video 1$)$. This rupture was located close to the left ventricular appendage and the circumflex artery (-Fig. 2A). Any attempts for direct closure of the myocardial rupture were unsuccessful due to fragility ("soft-cheese" consistence) of the myocardial muscle. A Dor, or modified Dor plasty, was not feasible in this case; therefore, the soft phlegmonlike myocardium was completely excised. Histopathological examination of the excised tissue revealed a myocardial infarction with the presence of multiple bacteria in coronary capillary vessels as well as bacterial imbibition of myocardial tissue (-Fig. 2B-D, - Fig. 3). For myocardial reconstruction, we used a $1.5 \times 1.5 \mathrm{~cm}$ large Dacron patch, which was placed on the endomyocardial side by eight single

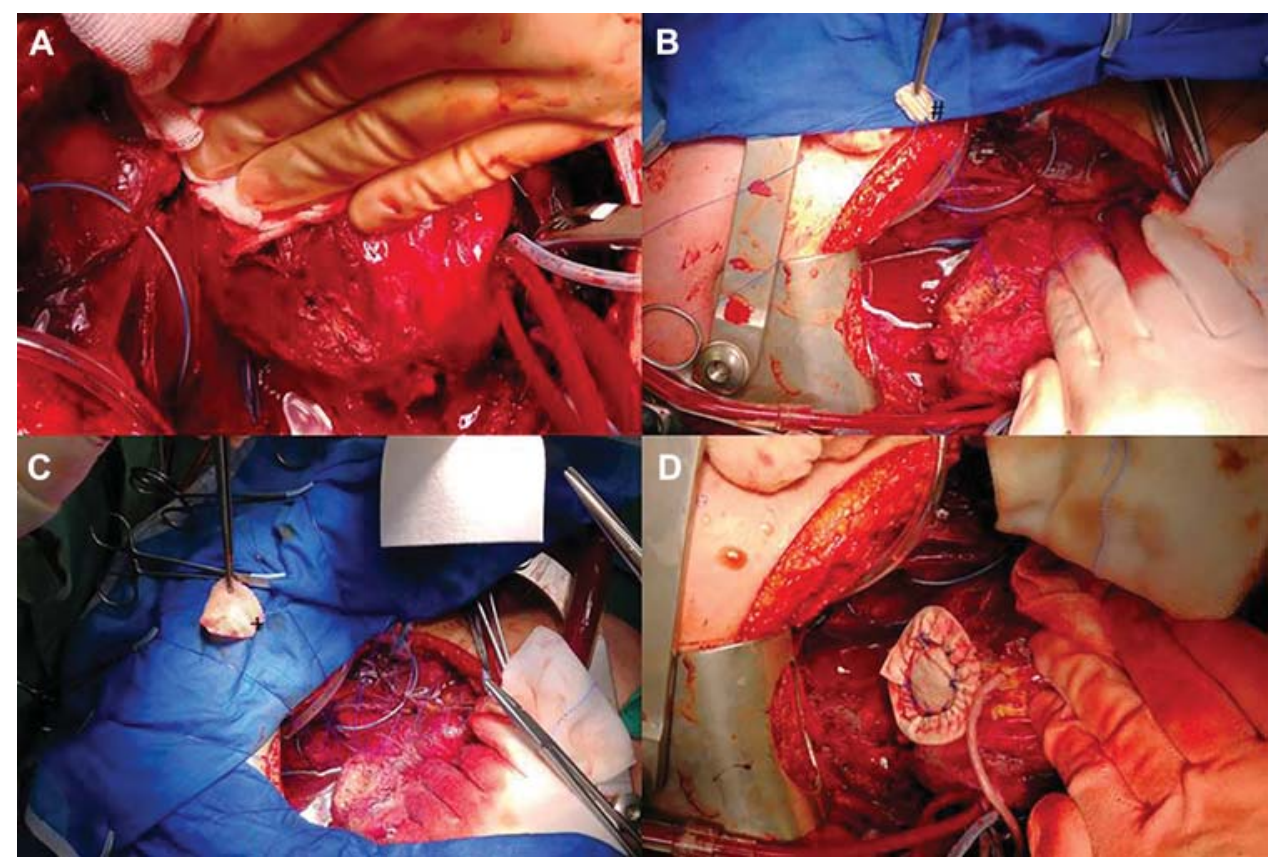

Fig. 2 Free left ventricular wall rupture in situ and reconstruction method. (A) Rupture of the left ventricular wall surrounded by necrotic tissue $\left(^{*}\right)$. (B) A $1.5 \times 1.5 \mathrm{~cm}$ large Dacron patch (\#) is placed on the endomyocardial side by eight single sutures. (C) The second Dacron patch (+) is placed to the outside of the myocardium and sutured to the initial eight Prolene single-stitch sutures. (D) An additional running suture placed around the second pericardial patch to prevent bleeding and the venous bypass graft to the circumflex artery. 


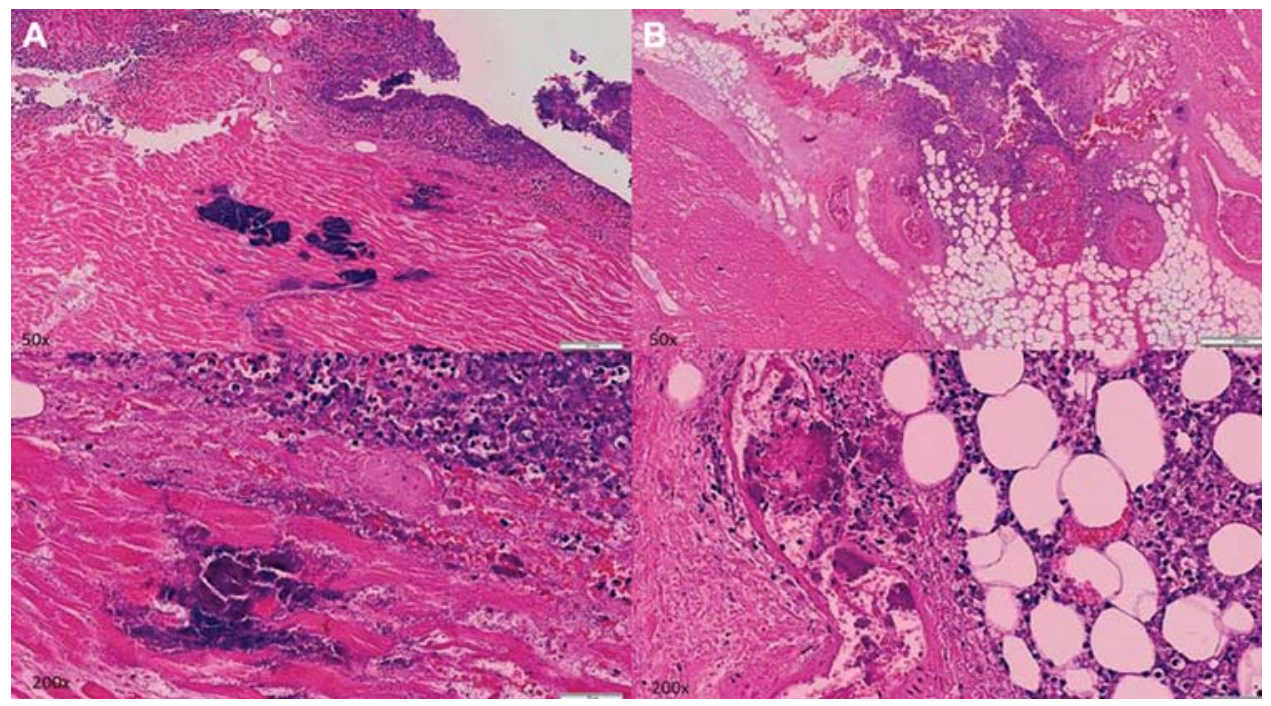

Fig. 3 Histopathology. (A) Hematoxylin and eosin (HE), $50 \times$ and $200 \times$ magnification, shows the cardiac phlegmon with necrotic myocardium colonized by bacteria and purulent inflammation. (B) HE, 50 $\times$ and $200 \times$ magnification, shows the cardiac phlegmon with necrotic myocardium and blood vessels with numerous thromboemboli containing bacteria.

sutures. Another larger Dacron patch was placed to the outside of the myocardium and sutured to the initial eight Prolene single stitch sutures ("empty-sandwich" technique). An additional running suture was placed around the second patch to prevent bleeding. Since the site of the perforation was close to the circumflex artery, additionally a venous bypass graft using the saphenous vein was performed to the posterolateral branch distal to the defect. BioGlue (CryoLife Inc., Kennesaw, GA) was used on the suture. After cardiac activity had been reestablished, no bleeding could be detected. To release mechanical stress from the left ventricle, the patient was placed on extracorporeal life support (ECLS) through the left femoral artery and vein. CPB was weaned and the patient was referred to the ICU after chest closure with mild-tomoderate catecholamine. ECLS was weaned 5 days after the initial operation. An abscess at the left foot had to be lanced. After 14 days of ICU stay postoperatively, the patient was referred to the administering peripheral hospital, where she recovered. Antibiotics were administered according to positive stated blood cultures for Staphylococcus aureus infection for 6 weeks postoperatively (clindamycin and flucloxacillin). Echocardiography finding after ECLS weaning revealed a left ventricular ejection fraction of $60 \%$ and a mild pericardial effusion without hemodynamic relevance. After 1 year, a pseudoaneurysm of the primarily implanted patch was detected and the patient needed a reoperation. Nonetheless, after successful aneurysm resection without complications and after a 7-day postoperative course, the patient was referred to rehabilitation and is now, half a year later, well at home.

\section{Conclusion}

Free left ventricular rupture caused by septic thromboembolism is a lethal complication of infectious endocarditis. To our knowledge, this is the first report on the development of a myocardial phlegmon with acute free wall rupture. Previously, phlegmonlike changes of the myocardial tissue have been reported by Osula et al. ${ }^{7}$ However, the authors described myocardial involvement in close proximity to skeptically destructed aortic and mitral valves in severe endocarditis. In our case, the cardiac phlegmon was localized in a free lateral wall of the left ventricle and was probably triggered by a septic coronary embolization. In contrast to all previously reported cases of myocardial rupture due to abscess and infectious endocarditis, to our knowledge, this is the first report describing a true phlegmon with myocardial rupture with a favorable outcome.

There are some issues to be addressed in our case. The finding of a hematopericardium should lead to immediate surgery in case of an endocarditis. After the initial operation and the closure of the small perforations at the base of the aorta, we assumed that this was the pathological mechanism for the pericardial effusion. We did not perform deairing through the apex and left the heart in place during the operation. We might have recognized the beginning of the left ventricular rupture during such a maneuver. However, albeit we usually perform deairing through the apex, we refrained from it in this particular case due to the adhesions caused by the inflammatory pericardium. Furthermore, a continuous $\mathrm{CO}_{2}$ insufflation has been performed during open heart surgery. We implanted a mechanical valve during the initial operation. It was the choice, made after consultation with our patient, for her age, not suffering from other comorbidities, and subsequently long life expectancy. However, after the second operation and with the patient on ECLS support, keeping the valve functional and preventing valve thrombosis was challenging.

A large defect of the myocardium in an otherwise healthy myocardium with a normal LVEDD (left ventricular enddiastolic diameter) can be a challenge. Classical approaches might lead to a significant reduction of the cardiac cavity and can lead to a significant loss of contractility of the heart. ${ }^{8}$ Using our technique, even larger defects can be replaced, without significant reduction of the left ventricular cavity. 
Although coronary artery thromboembolism and myocardial infarction are well-known complications of infective endocarditis, the finding of a free myocardial rupture remains a complication most often diagnosed by the pathologies postmortem. ${ }^{7}$ We need to distinguish between an aneurysm developed by direct extension of infection from septic valve vegetation into the underlying myocardium and this case of septic embolism causing a myocardial phlegmon and subsequent rupture. ${ }^{4}$

In summary, this is a rare case of a ventricular wall rupture in the context of a cardiac phlegmon due to septic coronary embolization.

\section{Video 1}

Free ventricular rupture after re-sternotomy. Online content including video sequences viewable at: https://www.thieme-connect.com/products/ ejournals/html/10.1055/s-0040-1716391.

\section{References}

1 Mostaghim AS, Lo HYA, Khardori N. A retrospective epidemiologic study to define risk factors, microbiology, and clinical outcomes of infective endocarditis in a large tertiary-care teaching hospital. SAGE Open Med 2017;5:2050312117741772

2 Toyoda N, Chikwe J, Itagaki S, Gelijns AC, Adams DH, Egorova NN. Trends in infective endocarditis in California and New York State, 1998-2013. JAMA 2017;317(16):1652-1660

3 Pant S, Patel NJ, Deshmukh A, et al. . Trends in infective endocarditis incidence, microbiology, and valve replacement in the United States from 2000 to 2011. J Am Coll Cardiol 2015;65 (19):2070-2076

4 Tayama E, Chihara S, Fukunaga S, Akashi H, Aoyagi S, Mizoguchi K. Embolic myocardial infarction and left ventricular rupture due to mitral valve endocarditis. Ann Thorac Cardiovasc Surg 2007;13 (03):206-208

5 Dickens P, Ho J, Wong KY. Sudden death from ruptured septic myocardial infarct in an intravenous drug addict. Forensic Sci Int 1995;75(01):67-72

6 Habib G, Lancellotti P, Antunes MJ, et al. . 2015 ESC Guidelines for the management of infective endocarditis: The Task Force for the Management of Infective Endocarditis of the European Society of Cardiology (ESC). Endorsed by: European Association for Cardio-Thoracic Surgery (EACTS), the European Association of Nuclear Medicine (EANM). Eur Heart J 2015;36(44): 3075-3128

7 Osula S, Lowe R, Perry RA. Cardiac rupture caused by Staphylococcus aureus septicaemia and pericarditis: an incidental finding. Heart 2001;85(03):E4

8 Schoen FJ, Levy RJ. Calcification of tissue heart valve substitutes: progress toward understanding and prevention. Ann Thorac Surg 2005;79(03):1072-1080 